\title{
Alterstice
}

Revue internationale de la recherche interculturelle

International Journal of Intercultural Research

Revista International de la Investigacion Intercultural

\section{Cultural development of mathematical ideas, de Geoffrey Saxe}

\section{Pierre R. Dasen}

Volume 3, numéro 1, 2013

URI : https://id.erudit.org/iderudit/1077502ar

DOI : https://doi.org/10.7202/1077502ar

Aller au sommaire du numéro

Éditeur(s)

Alterstice

ISSN

1923-919X (numérique)

Découvrir la revue

Citer ce compte rendu

Dasen, P. R. (2013). Compte rendu de [Cultural development of mathematical ideas, de Geoffrey Saxe]. Alterstice, 3(1), 93-96.

https://doi.org/10.7202/1077502ar d'utilisation que vous pouvez consulter en ligne.

https://apropos.erudit.org/fr/usagers/politique-dutilisation/ 


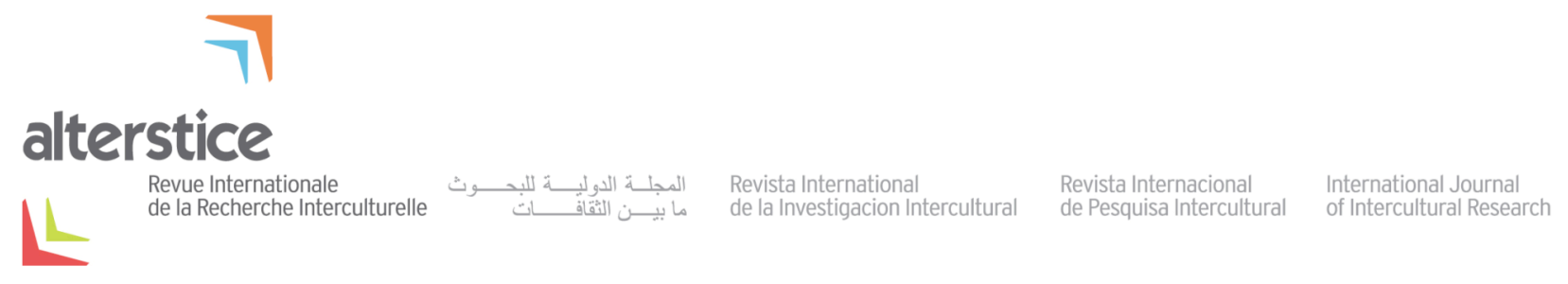

NOTE DE LECTURE

\title{
Cultural development of mathematical ideas, de Geoffrey Saxe
}

\author{
Pierre R. Dasen ${ }^{1}$
}

\author{
Rattachement de l'auteur \\ ${ }^{1}$ Université de Genève, Genève, Suisse
}

\section{Correspondance}

pierre.dasen@unige.ch

\section{Références de l'ouvrage :}

Saxe, G. (2012). Cultural development of mathematical ideas. Papua New Guinea Studies. Cambridge : Cambridge University Press, 393 p.

\section{Pour citer cet article :}

Dasen, P. (2013). Cultural development of mathematical ideas [note de lecture]. Alterstice, 3(1), 93-96.

\begin{abstract}
Les recherches de Geoffrey Saxe en Papouasie-Nouvelle-Guinée, dans les années 1978-1980, ont représenté pendant longtemps ce qu'on faisait de mieux dans les études interculturelles comparatives de la théorie de Piaget. Saxe étudiait, en particulier, comment les enfants oksapmin, dans les montagnes au centre du pays, apprenaient à maîtriser un système numérique traditionnel, faisant usage uniquement de 27 parties corporelles: à partir du pouce de la main droite comme équivalent du chiffre 1 (mais sans lexèmes particuliers pour les chiffres), on compte les cinq doigts, puis des parties du corps sur le bras (poignet $=6$, avant-bras $=7$, coude $=8$, etc.), le cou et la tête, puis l'autre bras jusqu'au petit doigt de la main gauche, qui représente 27. On ajoute le suffixe -bai pour indiquer qu'il s'agit d'une valeur cardinale, et à partir de l'oeil gauche le préfixe -tan pour indiquer la partie gauche du corps. Arrivé au petit doigt, il était habituel de s'arrêter en disant « tit fu » (le comptage est terminé). II est aussi possible de compter plus loin en revenant sur la même séquence, mais en pratique, on ne compte guère au-delà de 27.
\end{abstract}

Comment les enfants oksapmin acquièrent-ils la notion de nombre? Eh bien, de la même façon que les enfants genevois! et que, semble-t-il, tous les enfants du monde. Quand ils apprennent à compter, que ce soit avec la suite des nombres indo-arabes ou des parties du corps, en présence de deux collections égales d'objets disposés en lignes inégales, ils pensent qu'il y en a plus dans la ligne plus longue. Ce n'est qu'au stade suivant qu'ils peuvent utiliser le comptage pour se décentrer de l'aspect visuel trompeur. Le système oksapmin traditionnel pose d'ailleurs une petite difficulté supplémentaire, celle de reconnaître que deux parties du corps symétriques (comme l'oreille droite et l'oreille gauche) ne représentent pas la même valeur.

Les Oksapmin fabriquent des sacs en ficelle, dont ils mesurent la grandeur en y plongeant les bras, et en notant la partie du corps où se termine le sac. Mais pour le jeune enfant oksapmin, deux sacs arrivant au coude n'ont pas forcément la même grandeur si l'un est décalé par rapport à un autre. Ce n'est qu'au stade suivant qu'ils peuvent utiliser le système numérique pour la mesure d'une longueur. Saxe avait aussi inventé une épreuve spécifique à la 
situation, en demandant aux enfants si un sac qui leur arrivait à l'avant-bras arriverait au même endroit sur les bras d'un adulte. Certains jeunes enfants pensaient que le sac arriverait plus loin, p.ex. au coude, sur le bras plus long de leur père.

Ainsi, la méthodologie de Saxe était tout à fait exemplaire : il cherchait à vérifier (ou, scientifiquement, à falsifier) la théorie de Piaget, mais adaptait toute son étude aux particularités locales, non seulement en étudiant un système numérique exotique, mais en inventant des situations expérimentales appropriées, au lieu de traduire simplement les "épreuves piagétiennes" classiques comme le faisaient la plupart des autres chercheurs. Au niveau des résultats, il démontrait qu'en dépit de ces différences culturelles, et à l'exception d'un décalage dans le rythme de développement (l'âge auquel les différents sous-stades sont acquis), la construction du nombre et de la mesure suivaient un cheminement universel.

Dans une seconde partie de sa recherche de 1978-1980, Saxe examinait la possibilité d'adapter le système numérique traditionnel à des tâches nouvelles, telles que l'addition ou la soustraction. Traditionnellement, le système n'était jamais utilisé pour faire des calculs, seulement pour dénombrer. Mais dans les quelques boutiques vendant des produits de base (boutiques qui ont commencé à s'installer dans les années 1970), et en utilisant de l'argent plutôt que le troc, le calcul d'une somme était devenu fonctionnel, de même que la soustraction dans le calcul de la monnaie à rendre. Ainsi, les boutiquiers et les jeunes ayant passé quelques années à travailler endehors de la communauté dans les plantations, et ayant donc eu un contact plus étroit avec la monnaie, au contraire des adultes restés sur place, arrivaient à utiliser le système pour faire des calculs, en inventant différentes stratégies ad hoc (comme d'appeler une partie du corps par une autre). Essayez donc d'additionner poignet et coude (bien entendu, sans avoir le droit d'utiliser leur équivalent dans notre système décimal) : vous verrez que ce n'est pas si évident!

Fasciné par cette recherche originale, j'ai eu moi-même l'occasion de faire une étude dans une autre communauté de Papouasie-Nouvelle-Guinée, chez les Yupno (Wassmann et Dasen, 1994). Ces derniers utilisaient un système numérique basé également sur des parties du corps, mais de façon différente des Oksapmin: des lexèmes spécifiques existent pour un, deux, et trois; on commence à compter sur les doigts de la main gauche, puis la main droite, et ensuite les deux pieds, pour arriver à l'équivalent de 20 (un système très courant en PNG). Les jeunes s'arrêtaient là, mais les hommes âgés utilisaient des parties du corps pour aller au-delà, d'abord sur la tête (avec, à chaque fois, deux fois deux parties symétriques, comme les oreilles, puis une partie centrale, comme le nez), pour ensuite descendre le long du corps jusqu'au pénis (représentant 33). Les femmes, elles, disaient ne pas avoir le droit de compter (et prétendaient ne pas connaître le système). Grande déception quand nous avons voulu répliquer la partie développementale de la recherche de Saxe : les enfants Yupno ne connaissaient rien du système numérique traditionnel, mais apprenaient le système décimal à l'école.

Voilà donc pourquoi je m'intéressais tout particulièrement et personnellement à ce nouveau livre de G. Saxe. Mais il n'est pas nécessaire d'avoir été soi-même en Papouasie-Nouvelle-Guinée, ni même de s'intéresser particulièrement aux ethno-mathématiques, pour trouver de nombreux apports théoriques et méthodologiques originaux dans cet ouvrage. En plus, ce que je viens de résumer - parce que c'était pour moi très instructif à l'époque - ne représente qu'une infime partie du livre. En effet, G. Saxe est retourné sur le même terrain vingt ans plus tard, et c'est l'étude du changement économique et social, et l'impact de celui-ci sur l'utilisation du système numérique, qui forme la majeure partie de l'ouvrage. Là encore, G. Saxe fait oeuvre de pionnier. En effet, depuis quelque temps, les adeptes d'une théorie sociohistorique d'inspiration vygotskienne prônaient l'étude conjointe de la cognition et du changement social. Mais les exemples empiriques restaient rares sinon inexistants. Cette fois, nous avons enfin une étude complète et bien documentée utilisant ce nouveau paradigme de recherche.

En psychologie du développement, les études longitudinales sont essentielles, et pourtant peu nombreuses; il en est de même en anthropologie culturelle. II n'est pas si fréquent qu'un chercheur retourne plusieurs fois sur le même terrain pour documenter soi-même le changement social. Et pourtant, cela donne des études passionnantes, comme, par exemple, celle de Graburn (1982) sur l'impact de la télévision sur les adolescents inuits, où il n'a fallu que quelques années pour qu'ils adoptent des comportements violents. Ou encore l'étude exceptionnelle de Greenfield (2004) sur trois générations de jeunes filles mexicaines apprenant à tisser avec (ou sans) leurs mères.

Alterstice - Revue Internationale de la Recherche Interculturelle, vol. $3, n^{\circ} 1$ 
Greenfield démontre non seulement un changement cognitif en fonction du changement économique, mais aussi un impact sur les processus d'apprentissage.

Saxe décrit les changements socioéconomiques que la communauté oksapmin a subis sur une période historique relativement courte, p.ex. au niveau de l'introduction d'un système monétaire: d'abord la livre australienne (qui comprenait 20 shillings) dans les années 1960, puis le dollar australien de 1966 à 1975, et le kina (K) dès l'indépendance, où, par analogie à l'ancienne livre, une coupure du $2 \mathrm{~K}$ représente 20 pièces de 10 toea (parfois appelé " one shilling »). L'auteur analyse en détail comment les différentes parties du corps en sont venues à représenter différentes valeurs (p.ex. l'épaule pour $1 \mathrm{~K}$, soit 10 pièces de 10 toea), en oksapmin et en tok pisin (la lingua franca en Papouasie-Nouvelle-Guinée, utilisée à l'école tout comme l'anglais). Un autre chapitre est consacré aux changements dans la signification du terme fu, qui désignait d'abord un compte complet de 27 parties du corps, puis seulement jusqu'au coude gauche (20), pour désigner finalement, dans l'étude de 2001, le fait de doubler une quantité ou une partie du corps. Ces descriptions linguistiques détaillées dépassent peut-être parfois la patience du lecteur moyen, mais au moins l'information est disponible. C'est l'avantage d'une telle monographie, où les auteurs ne sont pas tenus de simplifier, comme c'est souvent le cas pour les articles dans les revues.

Une grande partie de l'étude s'intéresse à la scolarisation, qui a elle-même pris différentes formes au cours de I'histoire récente. Introduite à partir des années 1960 par les missionnaires pour des effectifs très restreints, puis à partir des années 1970 par le gouvernement colonial puis post-colonial pour des effectifs progressivement plus importants, l'école a été longtemps un corps étranger. Les enseignants venaient d'autres parties de PNG, ne parlaient pas l'Oksapmin, et le curriculum dévalorisait les savoirs locaux. Une réforme en 1998 a introduit au contraire une école plus communautaire, des enseignants Oksapmin utilisant la langue vernaculaire dans les premiers degrés, et cherchant à valoriser la culture traditionnelle. II est intéressant de voir à quel point l'école, quelle qu'elle soit, est systématiquement en décalage avec la réalité sociale. En 1978-80, les élèves connaissaient de toute évidence le système numérique traditionnel, puisque des photos montrent qu'ils comptaient sur le corps, en cachette des enseignants qui, eux, ne connaissaient rien de ce système. Dans les écoles actuelles, on enseigne au contraire le système traditionnel aussi bien que le système décimal, mais en en faisant une synthèse parfois un peu bizarre. P.ex. les enseignants utilisent l'épaule (10) comme une base (une épaule, deux épaules, etc.). À l'école, on fait aussi des calculs avec des unités de 1, 2 et 5 toea, qui n'ont en fait pas été mis en circulation dans la région.

L'intérêt principal de l'ouvrage, pour la plupart des lecteurs, sera sans aucun doute non pas dans le détail ethnographique, mais dans la conception méthodologique d'une telle étude. II s'agit vraiment d'une combinaison exemplaire d'anthropologie et de psychologie. On préconise souvent la recherche interdisciplinaire, mais là encore, les exemples vraiment convaincants sont relativement rares. En voilà pourtant un! Geoffrey Saxe, au départ psychologue se spécialisant dans le développement cognitif, devient tour à tour ethnologue, historien, linguiste et chercheur en sciences de l'éducation. Dans ses choix méthodologiques, il y a aussi le principe d'étudier systématiquement différents segments de la population : des enfants, scolarisés ou non, de jeunes adultes avec des expériences différentes (en particulier de contact avec le système économique plus large), des vieux qui connaissent bien la tradition, etc. Cela permet de combiner une approche sociologique transversale avec la perspective historique longitudinale.

L'auteur met sa recherche en perspective théorique en combinant ainsi la microgenèse, l'ontogenèse et la sociogenèse. Par analogie avec la théorie de l'évolution en biologie, il formule sa synthèse en termes de correspondances entre la forme (de la représentation d'un système numérique) et la fonction (les problèmes cognitifs à résoudre) dans des niches évolutives (différentes situations historiques, p.ex. liées à l'introduction du système monétaire). Personnellement, je regrette un peu tout ce qui peut suggérer un réductionnisme, qui caractérise déjà bien trop souvent la psychologie actuelle, mais l'intérêt théorique est dans le dépassement d'une étude purement intra-individuelle (des processus cognitifs) vers la prise en compte des formes culturelles de représentations et des fonctions qu'elles remplissent dans des pratiques collectives.

L'apport réellement original de ce livre me semble être dans la méthodologie. II s'agit là d'un changement de paradigme, dont l'intérêt va bien au-delà d'une étude monographique d'un sujet pointu dans une population exotique. Ainsi, l'ouvrage devrait intéresser un large public, au-delà de l'anthropologie culturelle et de la 
psychologie interculturelle comparée; il ravira, en particulier, tous ceux qui -- comme moi -- trouvaient l'approche constructiviste sociohistorique potentiellement intéressante, mais étaient un peu déçus par sa mise en pratique empirique.

On peut s'étonner du prix élevé fixé par Cambridge University Press : actuellement 60 f ou $99 \$$ US (78\$ US en format e-book). Bien entendu, il y a de très nombreux graphiques et photos dans le livre, ce qui augmente le coût de production, et il y a sans doute un marché assez restreint pour une telle monographie de recherche, mais tout de même : voilà un livre qui devrait avoir une distribution mondiale, y compris dans le monde " majoritaire " (les pays du Sud, ou " en développement », ou pauvres). Or quel est l'individu ou même la bibliothèque, en PapouasieNouvelle-Guinée ou ailleurs, qui pourra se permettre une telle dépense?

\section{Références bibliographiques}

Graburn, N. (1982). Television and the Canadian Inuit. Etudes/Inuit/Studies, 6, 7-17.

Greenfield, P. (2004). Weaving generations together. Santa Fe, NM : SAR Press.

Wassmann, J. et Dasen, P. (1994). Yupno number system and counting. Journal of Cross-Cultural Psychology, 25, 7894. 